\title{
Relay Selection in Relay-Assisted Free Space Optical Systems
}

\author{
Nestor D. Chatzidiamantis*, Diomidis S. Michalopoulos ${ }^{\dagger}$, Emmanouil E. Kriezis*, George K. Karagiannidis* \\ and Robert Schober ${ }^{\dagger}$ \\ *Department of Electrical \& Computer Engineering, Aristotle University of Thessaloniki, Thessaloniki, Greece, Emails: \{nestoras, mkriezis, geokarag\}@auth.gr \\ ${ }^{\dagger}$ Department of Electrical \& Computer Engineering, University of British Columbia, Vancouver, Canada, Emails: \{dio,rschober $@ @ e c e . u b c . c a$
}

\begin{abstract}
We investigate transmission protocols for relayassisted free-space optical (FSO) systems, when multiple parallel relays are employed and there is no direct link between the source and the destination. As an alternative to all-active FSO relaying, where all the available relays transmit jointly, we propose a scheme which selects only a single relay to participate in the communication between the source and the destination in each transmission slot, based on the channel state information (CSI) obtained from all FSO links. Thus, the need for synchronizing the relays' transmission is avoided, while the slowly varying nature of the atmospheric channel is exploited. For both relay selection and all-active relaying, novel analytical closed-form expressions for their outage performance are derived, assuming the GammaGamma channel model. Numerical results are provided for equal and non-equal length FSO links, which clearly demonstrate the significant performance gains offered by relay selection compared to all-active relaying.
\end{abstract}

\section{INTRODUCTION}

The constant need for higher data rates in support of highspeed applications has led to the development of the Free Space Optical (FSO) communication technology. Operating at unlicensed optical frequencies, FSO systems offer the potential of broadband capacity at low cost [1], and therefore, they present an attractive remedy for the "last-mile" problem. However, despite their major advantages, the widespread deployment of FSO systems is hampered by major impairments, which have their origin in the propagation of the optical signals through the atmosphere. Rain, fog and atmospheric turbulence are some of the major atmospheric phenomena that cause attenuation and rapid fluctuations in the received power of FSO signals, thereby increasing the error rate and severely degrading the overall performance [2].

In the past, several techniques have been applied in FSO systems for mitigating the degrading effects of the atmospheric channel, including error control coding in conjunction with interleaving [3], multiple-symbol detection [4], and spatial diversity [5]-[7]. Among those, spatial diversity, which is realized by deploying multiple transmit and/or receive apertures, has been particularly attractive, since it offers significant performance gains by introducing additional degrees of freedom in the spatial dimension. Thus, numerous FSO systems with multiple co-located transmit and/or receive apertures, referred as Multiple-Input Multiple-Output (MIMO) FSO systems, have been proposed in the technical literature [5]-[7]. However, in practice, MIMO FSO systems may not always be able to offer the gains promised by theory. This happens in cases where the assumption that all the links of the MIMO FSO system are affected by independent channel fading becomes invalid [7]. Furthermore, since both channel's path loss and fading statistics are distance-dependent, a large number of transmit and/or receive apertures is required in long-range links in order to achieve the desired performance gains, thus increasing the complexity of MIMO FSO systems.

In order to overcome such limitations, relay-assisted communication has been recently introduced in FSO systems as an alternative way to achieve spatial diversity [8]-[11]. The main idea lies in the fact that, by employing multiple relay nodes with line-of-sight (LOS) to both the source and the destination, a virtual multiple-aperture system is created, often referred as cooperative diversity system, even if there is no LOS between the source and the destination. The most seminal work in relay-assisted FSO systems was presented in [8], where various relaying configurations (cooperative diversity and multihop) have been investigated under the assumption of a lognormal channel model. Subsequently, several coding schemes for 3-way cooperative diversity FSO configurations with a single relay and a direct link between the source and the destination, have been proposed in [9], while the performance of such systems has been investigated in [10] and [11] assuming amplify-and-forward and decode-and-forward relaying strategies, respectively. It is emphasized that in all these previous works, all the available relays participated in the communication between the source and the destination, requiring perfect synchronization between the relays in order for the FSO signals to simultaneously arrive at the destination.

In view of the above, we present an alternative transmission protocol which can be used in relay-assisted FSO systems with no LOS between the source and the destination. Capitalizing on the fact that for the signaling rates of interest, the atmospheric channel does not vary within one packet and, thus, channel state information (CSI) can be easily obtained for all involved links by the source, the presented protocol selects only a single relay to take part in the communication in every transmission slot; the relay that maximizes an appropriately defined metric. Thus, the requirement of synchronization between the relays is avoided. Assuming the versatile GammaGamma channel model [2], as well as decode-and-forward relay nodes, we derive novel closed-form analytical expres- 
sions for the outage performance of the presented transmission scheme. In addition, we extend the outage analysis of the scheme where all the available relays are activated, originally proposed in [8], to the case of the Gamma-Gamma channel model.

The remainder of the paper is organized as follows. In Section II, the system model is described, providing details for the signal model in each FSO link, as well as the mode of operation of the considered relaying protocols. An analysis for the outage performance of both relaying protocols under investigation is provided on Section III, where closed-form analytical expressions are derived, while numerical results for various relay-assisted FSO architectures are presented in Section IV. Finally, concluding remarks are provided in Section V.

\section{SySTEM MODEL}

The system model under consideration is depicted in Fig. 1. In particular, we consider an intensity-modulation direct detection (IM/DD) FSO system without LOS between the source, $S$, and the destination, $D$, and the communication between these two terminals is achieved with the aid of multiple relays, denoted by $R_{i}, i \in\{1, \ldots, N\}$. The source node is equipped with a multiple-aperture transmitter, with each of the apertures pointing in the direction of the corresponding relay, and an optical switch $^{1}$, which either allows the transmission from all the transmit apertures or selects the direction of transmission by switching between the transmit apertures.

The presence of a large field-of-view (FOV) detector at the destination is assumed allowing for the simultaneous detection of the optical signals transmitted from each relay. Moreover, all optical transmitters are equipped with optical amplifiers that adjust the optical power transmitted in each link, while the relaying terminals operate in the threshold-based decodeand-forward (DF) mode; that is, they fully decode the received signal and retransmit it to the destination, only if the signal-tonoise ratio (SNR) of the receiving FSO link exceeds a given decoding threshold. Finally, throughout this paper, we assume that binary pulse position modulation (BPPM) is employed.

\section{A. Signal and Channel Model}

For an FSO link connecting two terminals $A$ and $B$, the received optical signal at the photodetector of $B$ is given by

$$
\mathbf{r}_{B}=\left[\begin{array}{c}
r^{s} \\
r^{n}
\end{array}\right]=\left[\begin{array}{c}
\eta T_{b}\left(\rho_{A B} P_{t} h_{A B}+P_{b}\right)+n^{s} \\
\eta T_{b} P_{b}+n^{n}
\end{array}\right]
$$

where $r^{s}$ and $r^{n}$ represent the signal and the non-signal slots of the BPPM symbol respectively, while $\rho_{A B} P_{t}$ and $P_{b}$ denote the average optical signal power transmitted from $A$ and the background radiation incident on the photodetector of $B$, respectively. Furthermore, $\rho_{A B}$ represents the percentage of the total optical power $P_{t}$ allocated to the FSO link between terminals $A$ and $B, h_{A B}$ is the channel gain of the link, $\eta$ is the photodetector's responsivity, $T_{b}$ is the duration of the

\footnotetext{
${ }^{1}$ Optical switches can be implemented with either spatial light modulators (SLM) [12, Ch. (27)] or optical MEMS devices [13].
}

signal and non-signal slots, and, $n^{s}$ and $n^{n}$ are the additive noise terms in signal and non-signal slots, respectively. Since background-noise limited receivers are assumed, where background noise is dominant compared to other noise components (such as thermal, signal dependent, and dark noise) [5], the noise terms can be modeled as additive white Gaussian noise, with zero mean and variance $\sigma_{n}^{2}=\frac{N_{0}}{2}$. After removing the constant bias $\eta T_{b} P_{b}$ from both slots, the instantaneous SNR of the link can be defined as [8]

$$
\gamma_{A B}=\frac{\eta^{2} \rho_{A B}^{2} T_{b}^{2} P_{t}^{2} h_{A B}^{2}}{N_{0}} .
$$

Due to atmospheric effects, the channel gain of the FSO link under consideration can be modeled as

$$
h_{A B}=\bar{h}_{A B} \tilde{h}_{A B}
$$

where $\bar{h}_{A B}$ accounts for path loss due to weather effects and geometric spread loss and $\tilde{h}_{A B}$ represents irradiance fluctuations caused by atmospheric turbulence. Both $\bar{h}_{A B}$ and $\tilde{h}_{A B}$ are time-variant, yet at very different time scales. The path loss coefficient varies on the order of hours while turbulence induced fading varies on the order of 1-100 ms [5]. Thus, taking into consideration the signaling rates of interest, which range from hundreds to thousands of Mbps, the channel gain can be considered as constant over a given transmission slot, which consists of hundreds of thousands (or even millions) of consecutive symbols.

The path loss coefficient can be calculated by combining the Beer Lambert's law [2] with the geometric loss formula [1, pp. 44], yielding

$$
\bar{h}_{A B}=\frac{D_{R}^{2}}{\left(D_{T}+\theta_{T} d_{A B}\right)^{2}} \exp \left(-v d_{A B}\right)
$$

where $D_{R}$ and $D_{T}$ are the receiver and transmitter aperture diameter, respectively; $\theta_{T}$ is the optical beam's divergence angle (in $m \mathrm{rad}$ ), $d_{A B}$ is the link's distance (in $\mathrm{km}$ ), and $v$ is the weather dependent attenuation coefficient (in $1 / \mathrm{km}$ ).

Under a wide range of atmospheric conditions, turbulence induced fading is statistically described by the well known Gamma-Gamma distribution [2]. The corresponding probability density function (pdf) of this model is given by

$$
\begin{aligned}
& f_{\tilde{h}_{A B}}(x)=\frac{2\left(\alpha_{A B} \beta_{A B}\right)^{\frac{\alpha_{A B}+\beta_{A B}}{2}}}{\Gamma\left(\alpha_{A B}\right)} \Gamma_{\left(\beta_{A B}\right)} \frac{\alpha_{A B}+\beta}{2}-1 \\
& \times K_{\alpha_{A B}-\beta_{A B}}\left(2 \sqrt{\alpha_{A B} \beta_{A B} x}\right)
\end{aligned}
$$

where $\Gamma(\cdot)$ is the Gamma function [14, Eq. (8.310)] and $K_{\nu}(\cdot)$ is the $\nu$ th order modified Bessel function of the second kind [14, Eq. (8.432/9)]. Further, $\alpha_{A B}$ and $\beta_{A B}$ are parameters related to the effective atmospheric conditions via [2]

$$
\alpha_{A B}=\left[\exp \left(\frac{0.49 \sigma_{R}^{2}}{\left(1+1.11 \sigma_{R}^{\frac{12}{5}}\right)^{\frac{7}{6}}}\right)-1\right]^{-1}
$$




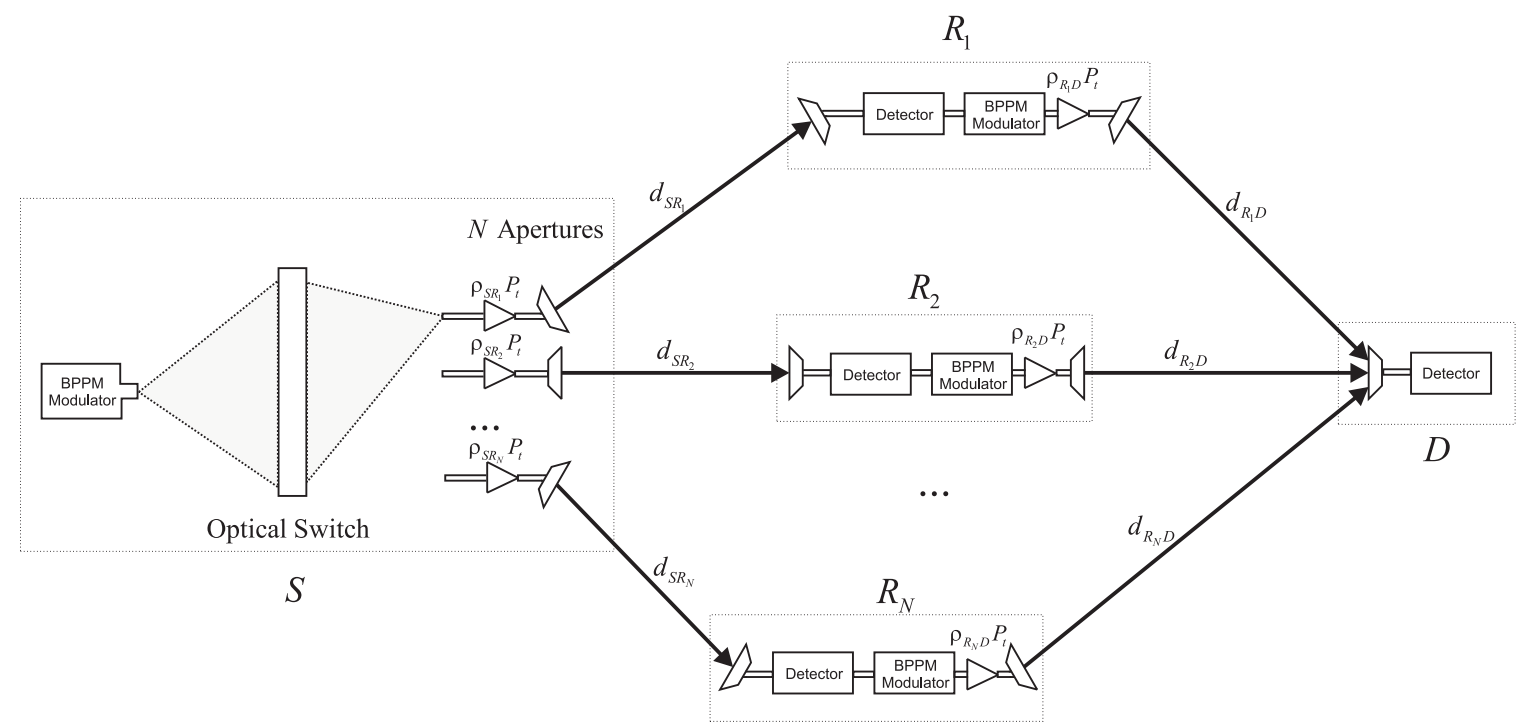

Fig. 1. The relay-assisted FSO system under consideration.

and

$$
\beta_{A B}=\left[\exp \left(\frac{0.51 \sigma_{R}^{2}}{\left(1+0.69 \sigma_{R}^{\frac{12}{5}}\right)^{\frac{7}{6}}}\right)-1\right]^{-1}
$$

with $\sigma_{R}^{2}=1.23 C_{n}^{2}\left(\frac{2 \pi}{\lambda}\right)^{\frac{7}{6}} d_{A B}^{\frac{11}{6}}$ being the Rytov variance, $C_{n}^{2}$ being the weather dependent index of refraction structure parameter, and $\lambda$ representing the wavelength of the optical carrier.

\section{B. Mode of Operation}

Throughout this work, two different cooperative relaying protocols are considered: the all-active protocol, originally presented in [8], and the select-max protocol.

1) All-active: In this relaying scheme, the source activates all relays and the total power is equally divided among all available FSO links. Since the relay nodes operate in the DF mode, only relays that successfully decode the received optical signal, remodulate the intensity of the optical carrier and forward the information to the destination. At the destination, owing to the presence of a large FOV aperture, aperture averaging occurs [6] and all the received optical signals are added. Hence, assuming perfect synchronization, the output of the combiner can be expressed as

$$
\mathbf{r}_{D}=\left[\begin{array}{c}
\eta T_{b}\left(\sum_{m \in \mathbf{D}} \rho_{R_{m} D} P_{t} h_{R_{m} D}+P_{b}\right)+n^{s} \\
\eta T_{b} P_{b}+n^{n}
\end{array}\right]
$$

where $\mathbf{D}$ denotes the decoding set formed by the set of the relays that have successfully decode the signal. Since the total power is equally divided among to all available links, it follows that

$$
\rho_{S R_{m}}=\rho_{R_{m} D}=\frac{1}{2 N}, m=1, \ldots, N .
$$

The advantage of this scheme is that no CSI is required at either the transmitter or the receiver side, since the source transmits to all available relays, regardless of their channel gain. However, since it is assumed that all the signals arrive at the destination at the same time, this scheme requires the employment of accurate synchronization techniques, in order to account for the different propagation delays caused by the different link distances, resulting in a complexity increase.

2) Select-Max: This relaying protocol selects a single relay out of the set of $N$ available relays in each transmission slot. In particular, that relay which maximizes an appropriately defined metric which accounts for both the $S-R_{i}$ and $R_{i}-D$ links and reflects the quality of the $i$ th end-to-end path. Here, we adopt

$$
\gamma_{i}=\min \left(\gamma_{S R_{i}}, \gamma_{R_{i} D}\right)
$$

as the quality measure of the $i$ th end-to-end path, which is based on the minimum SNR of the path's intermediate links. Note that (10) represents an outage-based definition of the selection metric, in the sense that an outage on the $i$ th endto-end link occurs if $\gamma_{i}$ falls below the outage threshold SNR. Hence, the selection of the single relay that is activated in the select-max relaying protocol, $R_{b}$, will be based on the rule

$$
b=\underset{i \in\{1, \ldots N\}}{\operatorname{argmax}} \gamma_{i} .
$$

Since a single relay is activated in the select-max protocol, the total available optical power is equally divided between the $S-R_{b}$ and $R_{b}-D$ links, i.e.,

$$
\rho_{S R_{b}}=\rho_{R_{b} D}=\frac{1}{2}
$$

and in the case that $R_{b}$ has successfully decoded its received optical signal, i.e., $b \in \mathbf{D}$, the signal at the destination can be expressed as

$$
\mathbf{r}_{D}=\left[\begin{array}{c}
\eta T_{b}\left(\rho_{R_{b} D} P_{t} h_{R_{b} D}+P_{b}\right)+n^{s} \\
\eta T_{b} P_{b}+n^{n}
\end{array}\right] .
$$


This relaying scheme requires CSI of all the available $S-R_{i}$ and $R_{i}-D$ FSO links at the transmitter in order to perform the selection process. This can be easily achieved by some signalling process that takes advantage of the slowlyvarying nature of the FSO channel and reports the CSI to the transmitter through a reliable RF feedback channel. It is emphasized that since only one end-to-end path is activated in each transmission slot, only one signal arrives at the destination and thus no synchronization between relays is needed.

\section{OUtAge AnALYsis}

At a given transmission rate, $r_{0}$, the outage probability is defined as

$$
P_{\text {out }}\left(r_{0}\right)=\operatorname{Pr}\left\{C(\gamma)<r_{0}\right\}
$$

where $C(\cdot)$ is the instantaneous capacity, which is a function of the instantaneous SNR. Since $C(\cdot)$ is monotonically increasing with respect to $\gamma$, (14) can be equivalently rewritten as

$$
P_{\text {out }}\left(r_{0}\right)=\operatorname{Pr}\left\{\gamma<\gamma_{\text {th }}\right\}
$$

with $\gamma_{t h}=C^{-1}\left(r_{0}\right)$ denoting the threshold SNR. If the SNR, $\gamma_{i}$, drops below $\gamma_{t h}$, an outage occurs, implying that the signal cannot be decoded with arbitrarily low error probability at the receiver. Henceforth, it is assumed that the threshold SNR, $\gamma_{t h}$, is identical for all the links of the relaying system.

\section{A. Outage Probability of the Intermediate Links}

Since DF relaying is considered, an outage event in any of the intermediate links may lead to an outage of the overall relaying scheme. Therefore, the calculation of the outage probability of each intermediate link is considered as a building block for both relaying schemes under investigation, and is thus utilized for the evaluation of the end-to-end performance.

By combining (2) with (15), the outage probability of the FSO link between nodes $A$ and $B$ is defined as

$$
P_{\text {out }, A B}=\operatorname{Pr}\left\{\frac{\eta^{2} T_{b}^{2} \rho_{A B}^{2} P_{t}^{2} h_{A B}^{2}}{N_{0}}<\gamma_{t h}\right\}
$$

which can be equivalently rewritten as

$$
P_{\text {out }, A B}=\operatorname{Pr}\left\{\tilde{h}_{A B}<\frac{1}{\bar{h}_{A B} \rho_{A B} P_{M}}\right\}
$$

where $P_{M}$ is the power margin given by

$$
P_{M}=\frac{\eta T_{b} P_{t}}{\sqrt{N_{0} \gamma_{t h}}} .
$$

Using the cumulative density function (cdf) of the GammaGamma distribution [15, Eq. (7)], the outage probability of the FSO link between nodes $A$ and $B$ can be analytically evaluated for any $\alpha_{A B}$ and $\beta_{A B}$, yielding

$P_{\text {out }, A B}=\frac{1}{\Gamma\left(\alpha_{A B}\right) \Gamma\left(\beta_{A B}\right)} G_{1,3}^{2,1}\left[\frac{\frac{\alpha_{A B} \beta_{A B}}{h_{A B}}}{P_{M} \rho_{A B}} \mid \begin{array}{c}1 \\ \alpha_{A B}, \beta_{A B}, 0\end{array}\right]$

\section{B. Outage Probability of All-Active Relaying}

In this scheme an outage occurs when either the decoding set $\mathbf{D}$ is empty or the SNR of the multiple input single output link between the decoding relays and the destination falls below the outage threshold. Hence, the outage probability of this scheme can be expressed as [8, Eq. (30)]

$$
P_{\text {out }}=\sum_{n=1}^{2^{N}} \operatorname{Pr}\left\{\sum_{m \in S(n)} h_{R_{m} D}<\frac{2 N}{P_{M}}\right\} \operatorname{Pr}\{S(n)\}
$$

where $S(n)$ denotes the $n$th possible decoding set (the total number of decoding sets is equal to $2^{N}$ ) and $\operatorname{Pr}\{S(n)\}$ is the probability of event $\{\mathbf{D}=S(n)\}$. By observing that

$$
\begin{aligned}
\operatorname{Pr}\{S(n)\} & =\prod_{m \in S(n)} \operatorname{Pr}\left\{\gamma_{S R_{m}}>\gamma_{t h}\right\} \\
& \times \prod_{m \notin S(n)} \operatorname{Pr}\left\{\gamma_{S R_{m}}<\gamma_{t h}\right\}
\end{aligned}
$$

(20) can be rewritten as

$$
\begin{aligned}
& P_{\text {out }}=\sum_{n=1}^{2^{N}} \prod_{m \in S(n)}\left(1-\operatorname{Pr}\left\{h_{S R_{m}}>\frac{2 N}{P_{M}}\right\}\right) \\
& \times \prod_{m \notin S(n)} \operatorname{Pr}\left\{h_{S R_{m}}<\frac{2 N}{P_{M}}\right\} \operatorname{Pr}\left\{\sum_{m \in S(n)} h_{R_{m} D}<\frac{2 N}{P_{M}}\right\} .
\end{aligned}
$$

In order to evaluate (22), the cdf of the sum of nonidentical Gamma-Gamma variates that correspond to decoding set $S(n), h_{S(n)}=\sum_{m \in S(n)} h_{R_{m} D}$, needs to be derived first. However, to the best of the authors' knowledge, there are no closed-form analytical expressions for the exact distribution of the non-identical Gamma-Gamma sum. Therefore, the numerical method of [16, Eq. (9.186)], which is based on the moment generating function (MGF) approach, is applied, and thus, the cdf of $h_{S(n)}$, denoted as $F_{h_{S(n)}}(\cdot)$, is evaluated via

$$
\begin{aligned}
& F_{h_{S(n)}}(x)=\frac{2^{-K} \exp \left(\frac{A}{2}\right)}{x} \\
& \quad \times \sum_{k=1}^{K}\left(\begin{array}{c}
K \\
k
\end{array}\right)\left(\frac{1}{2} \operatorname{Re}\left\{\frac{\prod_{m \in S(n)}\left(\mathcal{M}_{R_{m} D}\left(-\frac{A}{2 x}\right)\right)}{\frac{A}{2 x}}\right\}\right. \\
& \left.+\sum_{l=1}^{L+k}(-1)^{l} \operatorname{Re}\left\{\frac{\prod_{m \in S(n)}\left(\mathcal{M}_{R_{m} D}\left(-\frac{A+j 2 \pi l}{2 x}\right)\right)}{\frac{A+j 2 \pi l}{2 x}}\right\}\right)
\end{aligned}
$$

where $\mathcal{M}_{R_{m} D}(\cdot)$ is the MGF of the channel gain of the $R_{m} D$ FSO link given by [17, Eq. (4)] and the parameters $A, K$, and $L$ are calculated based on the numerical error term obtained from [16, Eq. (9.187)].

Theorem 1: The outage probability of the all-active relaying protocol in Gamma-Gamma fading can be analytically evaluated by (24), which is given at the top of the next page.

where $G_{p, q}^{m, n}[\cdot]$ is the Meijer's $G$-function [14, Eq. (9.301)]. 


$$
\begin{aligned}
P_{\text {out }}= & \sum_{n=1}^{2^{N}} \prod_{m \in S(n)}\left(1-\frac{1}{\Gamma\left(\alpha_{S R_{m}}\right) \Gamma\left(\beta_{S R_{m}}\right)} G_{1,3}^{2,1}\left[\frac{\alpha_{S R_{m}} \beta_{S R_{m}} N}{2 P_{M} \bar{h}_{S R_{m}}} \mid \begin{array}{c}
1 \\
\alpha_{S R_{m}}, \beta_{S R_{m}}, 0
\end{array}\right]\right) \\
& \times \prod_{m \notin S(n)} \frac{1}{\Gamma\left(\alpha_{S R_{m}}\right) \Gamma\left(\beta_{S R_{m}}\right)} G_{1,3}^{2,1}\left[\left.\frac{\alpha_{S R_{m}} \beta_{S R_{m}} N}{2 \bar{h}_{S R_{m}} P_{M}}\right|_{\alpha_{S R_{m}}, \beta_{S R_{m}}, 0}\right] F_{h_{S(n)}}\left(\frac{2}{N P_{M}}\right)
\end{aligned}
$$

Proof: The proof follows straightforwardly by combining (22) with (19) and (23).

\section{Outage Probability of Select-Max Relaying}

In the select-max protocol a single relay out of $N$ relays is selected according to the selection rule in (11). Hence, the outage probability of the relaying scheme under consideration is given by

$$
P_{\text {out }}=P_{\text {out }}\left\{R_{1} \cap \ldots \cap R_{N}\right\}=\prod_{b=1}^{N} P_{\text {out }}\left\{R_{b}\right\}
$$

where $P_{\text {out }}\left\{R_{b}\right\}$ denotes the probability of outage when only relay $R_{b}$ is active. Given that $R_{b}$ is active, an outage occurs when either $R_{b}$ or $D$ cannot decode the information successfully, i.e.,

$$
P_{\text {out }}\left\{R_{b}\right\}=\operatorname{Pr}\left\{\left(\gamma_{S R_{b}}<\gamma_{t h}\right) \cup\left(\gamma_{R_{b} D}<\gamma_{t h}\right)\right\}
$$

Hence, by combining (25) with (26), the probability of outage of the select-max relaying scheme is obtained as

$$
\begin{aligned}
P_{\text {out }}=\prod_{b=1}^{N}(1 & -\left(1-\operatorname{Pr}\left\{\tilde{h}_{S R_{b}}<\frac{2}{\bar{h}_{S R_{b}} P_{M}}\right\}\right) \\
& \left.\times\left(1-\operatorname{Pr}\left\{\tilde{h}_{R_{b} D}<\frac{2}{\bar{h}_{R_{b} D} P_{M}}\right\}\right)\right)
\end{aligned}
$$

The following theorem provides an accurate analytical expression for performance evaluation of the select-max relaying scheme.

Theorem 2: The probability of outage of a relay-assisted FSO system that employs the select-max relaying protocol, when operating over Gamma-Gamma fading channels, is obtained by (28), which is given at the top of the next page.

Proof: The proof follows directly from (27), in conjunction with (19).

\section{NumericAl RESUlts AND Discussion}

In this section, we illustrate numerical results for the outage performance of the considered relaying protocols, using the derived analytical expressions. In the following, we consider a relay-assisted FSO system with $\lambda=1550 \mathrm{~nm}$ and diameters of $D_{R}=D_{T}=20 \mathrm{~cm}$ for the transmit and receive apertures. Furthermore, we assume clear weather conditions with visibility of $10 \mathrm{~km}$, which correspond to a weather-dependent attenuation coefficient of $v=0.1$ and an index of refraction structure parameter of $C_{n}^{2}=2 \times 10^{-14} \mathrm{~m}^{-\frac{2}{3}}$.

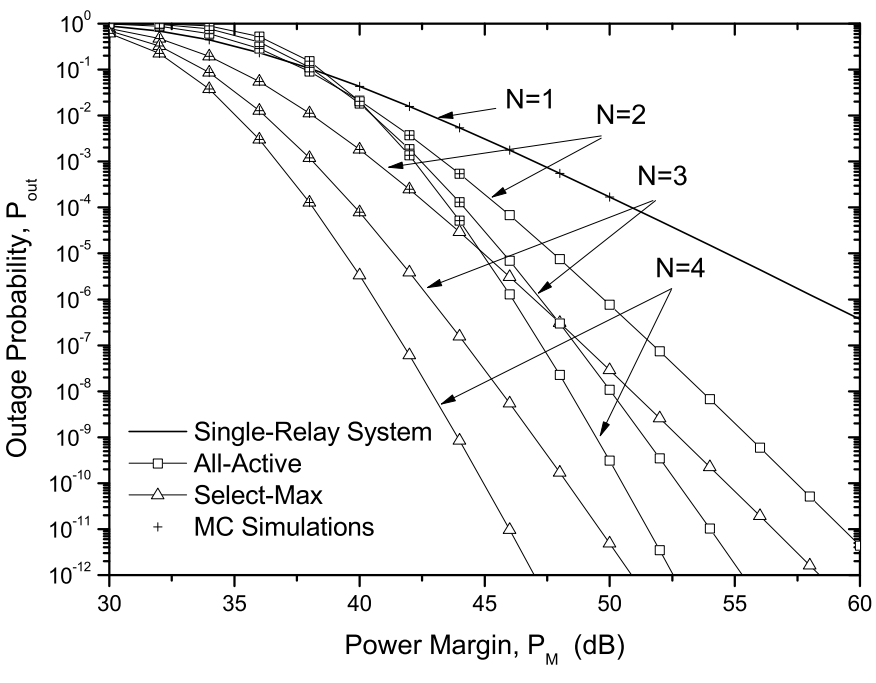

Fig. 2. Comparison of relaying protocols for a relay-assisted FSO system with $d_{S R_{i}}=d_{R_{i} D}=2 \mathrm{~km}, i \in\{1, \ldots, N\}$.

Fig. 2 depicts the outage performance of the presented relaying protocols for various numbers of relays, when the link distance is identical for all $S-R_{i}$ and $R_{i}-D$ links. Specifically, analytical results for the outage probability of the equidistant relay-assisted FSO system with a link distance of $2 \mathrm{~km}$ are plotted, as a function of the power margin for $N=2,3,4$ relays, using (24) and (28). As benchmarks, Monte Carlo (MC) simulation results and the performance of an FSO system with $N=1$, which is independent of the employed relaying protocol, are also illustrated in Fig. 2. As can be observed, there is an excellent match between simulation and analytical results for every value of $N$, verifying the presented theoretical analysis. Moreover, it is observed that the select-max relaying scheme has a better performance than the all-active scheme in every case examined $(2,4$, and $5 \mathrm{~dB}$ performance gains are observed for $N=2,3$, and 4 respectively). This result is intuitively pleasing, since the select-max protocol selects in each transmission slot the best end-to-end path out of the $N$ available paths and allocates the total available optical power only to this path. It should be noted that for the select-max protocol CSI of the transmission links is required, yet this can be easily obtained due to the slowly varying nature of the atmospheric channel. Fig. 3 depicts the outage performance of a relay-assisted FSO system employing the presented protocols and assuming different distances for each of the $S-R_{i}$ and $R_{i}-D$ FSO links. Specifically, two different system configurations are investigated: in the first system 


$$
\begin{aligned}
P_{\text {out }}= & \prod_{b=1}^{N}\left(1-\left(1-\frac{1}{\Gamma\left(\alpha_{S R_{b}}\right) \Gamma\left(\beta_{S R_{b}}\right)} G_{1,3}^{2,1}\left[\frac{2 \alpha_{S R_{b}} \beta_{S R_{b}}}{P_{M} \bar{h}_{S R_{b}}} \mid \begin{array}{c}
1 \\
\alpha_{S R_{b}}, \beta_{S R_{b}}, 0
\end{array}\right]\right)\right. \\
& \left.\times\left(1-\frac{1}{\Gamma\left(\alpha_{R_{b} D}\right) \Gamma\left(\beta_{R_{b} D}\right)} G_{1,3}^{2,1}\left[\frac{2 \alpha_{R_{b} D} \beta_{R_{b} D}}{P_{M} \bar{h}_{R_{b} D}} \mid \begin{array}{c}
1 \\
\alpha_{R_{b} D}, \beta_{R_{b} D}, 0
\end{array}\right]\right)\right)
\end{aligned}
$$

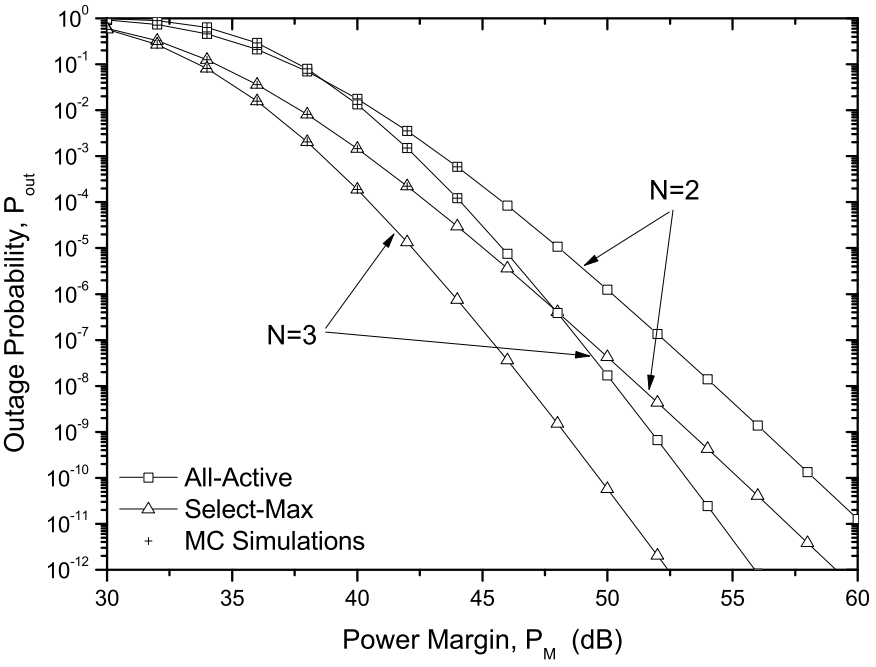

Fig. 3. Comparison of relaying protocols for different relay-assisted FSO configurations: $N=2, d_{S R}=\{2,1.5\}, d_{R D}=\{1,2.5\}$ (in $\mathrm{km}$ ) and $N=3, d_{S R}=\{2,1.5,1\}, d_{R D}=\{1,2.5,3\}$ (in km).

configuration, $N=2$ and the link distances are given by the vectors $d_{S R}=\{2,1.5\}$ and $d_{R D}=\{1,2.5\}$, with the elements of the vectors respresenting the distances (in $\mathrm{km}$ ) of the $S-R_{i}$ and $R_{i}-D$ links respectively, while in the second configuration $N=3$, and the link distances are given by $d_{S R}=\{2,1.5,1\}$ and $d_{R D}=\{1,2.5,3\}$. Fig. 3 reveals that the select-max relaying scheme offers significant performance gains compared to the all-active scheme, also for non-equal link distances. In particular, in the first configuration a gain of $2.5 \mathrm{~dB}$ compared to the all-active scheme is offered, while in the second configuration the offered gain is $3 \mathrm{~dB}$. Furthermore, simulation and analytical results again in excellent agreement.

\section{CONCLUSIONS}

We investigated transmission protocols for relay-assisted FSO systems without direct link between the source and the destination for the Gamma-Gamma channel model. An alternative to the all-active relaying scheme was proposed, which selects only a single relay to participate in the communication between the source and the destination in each transmission slot, based on the CSI obtained from all FSO links. Thus, the need for synchronizing the relays' transmission in order for the FSO signals to arrive simultaneously at the destination is avoided. The mode of operation of the proposed transmission method was presented, and its outage performance along with the performance of the all-active scheme were analyzed. Numerical results were provided for both equal and non-equal length FSO links, which clearly demonstrated the significant performance gains offered by relay selection compared to allactive relaying.

\section{REFERENCES}

[1] H. Willebrand and B. S. Ghuman, Free Space Optics: Enabling Optical Connectivity in Todays Networks. Sams Publishing, 2002.

[2] L. Andrews, R. L. Philips, and C. Y. Hopen, Laser Beam Scintillation with Applications. SPIE Press, 2001.

[3] X. Zhu and J. M. Kahn, "Performance Bounds for Coded Free-Space Optical Communications through Atmospheric Turbulence Channels," IEEE Trans. on Commun., vol. 51, pp. 1233-1239, Aug. 2003.

[4] M. L. B. Riediger, R. Schober, and L. Lampe, "Fast Multiple-Symbol Detection for Free-Space Optical Communications," IEEE Trans. on Commun., vol. 57, pp. 1119-1128, Apr. 2009.

[5] E. Lee and V. Chan, "Part 1: Optical Communication over the Clear Turbulent Atmospheric Channel Using Diversity," IEEE J. Sel. Areas Commun., vol. 22, pp. 71 896-1906, Nov. 2004.

[6] S. M. Navidpour and M. Uysal, "BER Performance of Free-Space Optical Transmission with Spatial Diversity," IEEE Trans. Wireless Commun., vol. 6, pp. 2813-2819, Aug. 2007.

[7] S. G. Wilson, M. Brandt-Pearce, Q. Cao, and J. H. Leveque, "Free-Space Optical MIMO Transmission with Q-ary PPM," IEEE Trans. Commun., vol. 53, pp. 1402-1412, Aug. 2005.

[8] M. Safari and M. Uysal, "Relay-Assisted Free-Space Optical Communication," IEEE Trans. on Wireless Commun., vol. 7, pp. 5441-5449, Dec. 2008.

[9] M. Kamiri and N. Nasiri-Kerari, "BER Analysis of Cooperative systems in Free-Space Optical Networks," IEEE/OSA J. Lightw. Techn., vol. 27, pp. 5639-5647, Dec. 2009.

[10] - "Free-Space Optical Communications via Optical Amplify-andForward Relaying," IEEE/OSA J. Lightw. Techn., vol. 29, pp. 242-248, Jan. 2011.

[11] C. Abou-Rjeily and A. Slim, "Cooperative Diversity for Free-Space Optical Communications: Transceiver Design and Performance Analysis," to be published in IEEE Trans. Commun.

[12] H. S. Nalwa, Handbook of Organic Electronics and Photonics. American Scientific Publishers, 2006.

[13] M. Yano, F. Yamagishi, and T. Tsuda, "Optical MEMS for Photonic Switching-Compact and Stable Optical Crossconnect Switches for Simple, Fast, and Flexible Wavelength Applications in Recent Photonic Networks," IEEE J. Sel. Topics Quantum Electron., vol. 11, pp. 383-194, 2005.

[14] I. S. Gradshteyn and I. M. Ryzhik, Table of Integrals, Series, and Products, 7th ed. Academic, 2007.

[15] T. A. Tsiftsis, "Performance of Heterodyne Wireless Optical Communication Systems over Gamma-Gamma Atmospheric Turbulence Channels," Electron. Lett., vol. 44, Feb. 2008.

[16] M. K. Simon and M. S. Alouini, Digital communications over Fading Channels. Wiley Interscience, 2005.

[17] P. S. Bithas, N. C. Sagias, P. T. Mathiopoulos, G. K. Karagiannidis, and A. A. Rontogiannis, "On the Performance Analysis of Digital Communications over Generalized-K Fading Channels," IEEE Commun. Lett., vol. 10, pp. 353-355, May 2006. 